\title{
Development and Validation of a Nomogram for Predicting Recurrence-Free Survival of Surgical Resected Retroperitoneal Liposarcoma
}

\author{
Aobo Zhuang' \\ Qian $\mathrm{Wu}^{\prime}$ \\ Hanxing Tong ${ }^{1,2}$ \\ Yong Zhang ${ }^{1,2}$ \\ Weiqi Lu ${ }^{1,2}$
}

'Department of General Surgery, South Hospital of Zhongshan Hospital/Shanghai Public Health Clinical Center, Fudan University, Shanghai, People's Republic of China; ${ }^{2}$ Department of General Surgery, Zhongshan Hospital, Fudan University,

Shanghai, People's Republic of China
Correspondence: Weiqi Lu; Yong Zhang Email lu.weiqi@zs-hospital.sh.cn; |3681971072@I63.com
Background and Objectives: Surgical treatment is still the mainstay of curative therapy for retroperitoneal liposarcoma (RLPS), but often recurs after surgical resection. We aimed to establish a nomogram for postoperative recurrence of RLPS based on the Asian population. Methods: Patients after surgical resection at the South Hospital of Zhongshan Hospital/ Shanghai Public Health Clinical Center between August 2011 and December 2020 were included. The enrolled patients are randomly divided into training set and test set according to the ratio of 7:3. Prognostic factors were chosen based on Akaike Information Criterion, and the nomogram was built based on Cox regression and then internally validated through calibration plots and concordance index (C-index).

Results: A total of 447 patients were included. Gender, age, presentation status, organ invasion and FNCLCC grade were used to build nomogram. The calibration plots showed that RFS predicted probabilities are identical to the actual RFS rates. The C-index of the nomogram was 0.703 (95\% CI $0.623-0.783)$ in the training set and 0.695 (95\% CI 0.565 0.825 ) in test set.

Conclusion: The nomogram we established can accurately predict postoperative recurrence of RLPS patients for Asian population.

Keywords: retroperitoneal soft tissue sarcoma, liposarcoma, recurrence, nomogram, surgery

\section{Introduction}

Retroperitoneal liposarcoma (RLPS) is a very rare malignant tumor that originates in the retroperitoneal mesenchymal tissue. Its incidence rate is only 2.5 per million population and accounts for $0.07 \%$ to $0.2 \%$ of all neoplasias. ${ }^{1}$ Nowadays, surgery is still the main and most effective treatment. ${ }^{2}$ However, it often recurs 6 to 24 months after surgery. ${ }^{3}$ The local recurrence rate of RLPS correlates with histologic subtype.

Studies show that the 5 years local recurrence rates about $50 \%$ for WDLPS and $80 \%$ for DDLPS. ${ }^{4}$ Nomogram, an emerging tool for prognostic assessment of cancer, has been widely used to predict the prognosis of retroperitoneal soft tissue sarcoma, ${ }^{4-6}$ but rarely for RLPS.

With a population of more than 4 billion, the Asia-Pacific region is the most diverse and fastest-growing region in the world. There are a number of reports of large cases of retroperitoneal liposarcoma postoperative recurrence in Western countries, ${ }^{7}$ but still only few reports based on large samples of Asian populations.

In this study, we intend to develop a nomogram to predict 1 year, 2 years and 5 years RFS rate in RLPS based on Asian patients. 


\section{Methods}

\section{Patients}

Four hundred and sixty-five consecutive patients with curative intent between August 2011 and December 2020 at the Sarcoma Center, General Surgery Department, South Hospital of the Zhongshan Hospital/Shanghai Public Health Clinical Center, Fudan University, Shanghai, China were included. The inclusion criteria were as follows: (1) the tumor is located in the retroperitoneum, (2) pathologically confirmed as liposarcoma, (3) complete clinical pathological information, (4) complete surgical resection (R0 or R1), (5) complete follow-up data. Eighteen patients lost to follow-up were excluded. Therefore, 447 consecutively enrolled RLPS patients were included in our study. This study was approved by the Ethics Committee of South Hospital of Zhongshan Hospital/Shanghai Public Health Clinical Center, the authorization number is 2020-S058-02, and was carried out following the Declaration of Helsinki.

\section{Clinicopathologic Evaluation}

We collected clinicopathological risk factors that may have an impact on prognosis, including gender, age, presentation status (primary or recurrence), chemotherapy, radiotherapy, tumor location (left or right), tumor size, organ invasion, histologic subtypes, FNCLCC grade and the number of resected organs.

For presentation status, patients with RLPS who undergo surgery for the first time are defined as primary disease, others are recurrent disease. The tumor location is divided into left and right, with RLPS originating from the left retroperitoneum (subdiaphragm, supra-iliac vessels, lateral axillary midline, and medial vertebral column), the right side is opposite of the left side.

In 2013, the World Health Organization classified liposarcoma into the following four pathological types: (1) well-differentiated liposarcoma (WDLPS), (2) dediferentiated liposarcoma (DDLPS), (3) myxoid/round cell liposarcoma (MLPS), and (4) pleomorphic liposarcoma (PLPS). ${ }^{8}$ WD/DDLPS is characterized by amplification of 12q13-15 (including the oncogenes MDM2 and CDK4); MLPS by translocation of FUS and DDIT3 (CHOP) genes; PLPS has no specific immunohistochemical or molecular genetic features, the presence of lipoblasts remains the sole diagnostic criterion. According to the French Federation of Centers for the Fight against
Cancer (FNCLCC) criteria, tumour were graded into I, II, and III. ${ }^{8}$

The postoperative follow-up is as follows: each followup requires clinical and imaging examination (CT or MRI of the chest, abdomen and pelvis). Follow-up every 3 months for the first 2 years, every 6 months after 2 years, and every year after 5 years. A new lesion diagnosed by imaging is defined as a disease recurrence. Recurrence-free survival is defined as the time interval from the date of surgery to the date of liposarcoma recurrence.

\section{Statistical Analysis}

Before we conduct data analysis, we randomly assign all patients to the training set and test set at a ratio of $7: 3$. Nomogram was built based on the training set and then internally validated on the test set.

Fisher's exact test or Pearson Chi-square was used to compare categorical variables such as gender, performance status, chemotherapy, radiotherapy, tumor location, organ invasion, histological subtype and FNCLCC grade. We use an independent sample $t$-test to compare continuous variables (age and tumor size). We calculated and compared RFS rates using Kaplan-Meier and Log rank tests. Given that the role of adjuvant therapy (chemotherapy/radiotherapy) in the treatment of RLPS is still controversial, and only a small number of patients in this study received adjuvant therapy. The status of chemotherapy and radiotherapy was only described in the baseline, with no further analysis.

As for variable selection, all clinicopathologic variables were included, and a backward procedure based on the Akaike Information Criterion (AIC) ${ }^{9}$ was applied. The development of the nomogram was based on a multivariate Cox model with the selected variable. Utilizing Harrell C's consistency index to assess discrimination. For calibration, the patients were divided into 3 subgroups based on the predicted survival probability. Calculate and plot the mean and $95 \%$ confidence interval of each subgroup.

Finally, according to the median score of the nomogram prediction score, we divided the patients into highrisk and low-risk groups.

All the tests were two-tailed and $\mathrm{P}<0.05$ considered statistically significance. All data were analyzed using SPSS 22.0 (SPSS Inc., Chicago, IL, USA) and R 4.0.4 (R Foundation for Statistical Computing, Vienna, Austria; http://www.r-project.org/). 


\section{Results}

\section{Baseline Patient Demographics}

Of all 447 patients with RLPS who met the criteria were included, the median follow-up time for the survivors $(\mathrm{n}=339)$ was 31.7 months (IQR 15.3-63.6). In all patients, $229(51.2 \%)$ suffer from recurrence. The median RFS was 23.6 (95\% CI 20.8-26.3) months. The 2 years and 5 years RFS rates were $47.3 \%$ (95\% CI 44.-50.1\%) and $25.1 \%$
(95\% CI 21.9-28.3\%), respectively. The baseline characteristics of the training set and the test set are basically the same (see Table 1 for details).

\section{Feature Selection}

Cox regression analysis began with the following clinical variables: gender, age, tumor size, presentation status, tumor location, organ invasion, histologic subtypes,

Table I Demographic, Clinicopathologic and Treatment Characteristics of All Patients, as Well as Patients in Training and Test Set

\begin{tabular}{|c|c|c|c|c|}
\hline Variables & Total $(n=447)$ & Training Set $(n=3 \mid 3)$ & Test Set $(n=134)$ & p value \\
\hline \multicolumn{5}{|l|}{ Gender } \\
\hline Male & 218 & 148 & 70 & 0.337 \\
\hline Female & 229 & 165 & 64 & \\
\hline Age (yr) [median (IQR)] & $55,44-66$ & $56,45-67$ & $54,44-64$ & 0.172 \\
\hline \multicolumn{5}{|l|}{ Presentation status } \\
\hline Primary & 153 & 104 & 49 & 0.495 \\
\hline Recurrence & 294 & 209 & 85 & \\
\hline Chemotherapy & & & & 0.437 \\
\hline Yes & 52 & 34 & 18 & \\
\hline No & 395 & 279 & 116 & \\
\hline Radiation therapy & & & & 0.695 \\
\hline Yes & 27 & 18 & 9 & \\
\hline No & 420 & 295 & 125 & \\
\hline \multicolumn{5}{|l|}{ Location } \\
\hline Left & 232 & 162 & 70 & 0.926 \\
\hline Right & 215 & $|5|$ & 64 & \\
\hline $\operatorname{Size}(\mathrm{cm})$ [median (IQR)] & $17,9-25$ & $17,9-25$ & $17,8-26$ & 0.735 \\
\hline \multicolumn{5}{|l|}{ Organ invasion } \\
\hline Yes & 377 & 265 & 112 & 0.773 \\
\hline No & 70 & 48 & 22 & \\
\hline \multicolumn{5}{|l|}{ Histologic subtypes } \\
\hline Well-diferentiated (WDLPS) & 208 & 152 & 56 & 0.362 \\
\hline Dediferentiated (DDLPS) & 187 & 128 & 59 & \\
\hline Myxoid/Round cell (MLPS) & 40 & 24 & 16 & \\
\hline Pleomorphic (PLPS) & 12 & 9 & 3 & \\
\hline \multicolumn{5}{|l|}{ FNCLCC } \\
\hline Grade I & 127 & 94 & 33 & 0.506 \\
\hline Grade 2 & 198 & 136 & 62 & \\
\hline Grade 3 & 122 & 83 & 39 & \\
\hline \multicolumn{5}{|l|}{ Number of resected organs } \\
\hline$\leq 1$ & 190 & 126 & 64 & 0.141 \\
\hline$>1$ & 257 & 187 & 70 & \\
\hline
\end{tabular}


FNCLCC grade and the number of resected organ. Backward step-wise selection was performed using a likelihood ratio test with AIC as the stopping rule. After that, gender, age, presentation status, organ invasion and FNCLCC were selected to build the RFS nomogram (Table 2).

\section{Development of the Nomogram}

A nomogram combining the above predictors is shown based on a multivariate Cox model.

The nomogram can be used to accurately predict the RFS rate of RLPS patients at 1 year, 2 years, and 5 years after surgery (Figure 1). Each indicator corresponds to a score in the nomogram. We add each score corresponding to the five risk factors to get a total score. The intersection of the vertical line of the total minute axis and the 1-, 2-, and 5-year recurrence-free survival axis is the probability of 1 year, 2 years, and 5 years of recurrencefree survival predicted by the model. In the nomogram, an increase in the total score stands for an increase in the risk of recurrence.

\section{Validation of the Nomogram}

Figure 2 demonstrates the calibration curve for training and test sets at 1, 2 and 5 years. The calibration curves of RFS showed optimal agreement between the predicted and observed RFS rate. The Harrell C-index for the nomogram was $0.703(95 \%$ CI $0.623-0.783)$ in the training set and $0.695(95 \%$ CI $0.565-0.825)$ in the test set.

\section{Risk Stratification for Liposarcoma Patients}

Finally, based on the median score, we divided the patients into a high-risk group and a low-risk group. The RFS curve of the two groups of patients is shown in Figure 3 $(\mathrm{p}<0.001)$.

\section{Discussion}

Retroperitoneal liposarcoma is a very rare disease with an extreme incidence. ${ }^{3}$ The only effective treatment for patients is surgery. ${ }^{10}$ Unfortunately, the probability of recurrence after surgery is extremely high. ${ }^{7}$ Several reports have showed that nomograms, as an emerging tool, perform better in predicting the overall survival and RFS for retroperitoneal sarcoma. ${ }^{5,11,12}$ The most famous retroperitoneal tumor prognostic nomogram prediction model was created by Gronchi et al in 2013. ${ }^{5}$ Three independent series of external verification confirmed that these nomograms are well calibrated and maintain good discrimination when applied to external cohorts. ${ }^{13,14}$ However, it did not establish a separate prediction model for liposarcoma. In 2006, the MSKCC group developed an LPS-specific postoperative nomogram that combined eight covariates to calculate the 5-year and 12-year disease-specific survival probabilities, with a concordance index of 0.827. . $^{4}$ While tumor depth (deep or superficial) is included in the nomogram prediction model, it relies on postoperative variables. Therefore, LPS specific postoperative nomogram is an inadequate preoperative counseling tool.

Table 2 Results of the Univariable Cox Models and Wald Test for Backward Selected Variables

\begin{tabular}{|c|c|c|c|c|}
\hline & \multicolumn{3}{|c|}{ cox Univariable } & \multirow{2}{*}{$\begin{array}{l}\text { Wald Test } \\
\text { p value }\end{array}$} \\
\hline & OR & $95 \% \mathrm{Cl}$ & p value & \\
\hline Gender (Male) & 1.142 & $0.779-1.675$ & 0.495 & 0.0219 \\
\hline Age & 0.997 & $0.981-1.014$ & $0.76 \mathrm{I}$ & 0.0735 \\
\hline Presentation status (Primary) & 2.524 & $1.597-3.990$ & $<0.001$ & $<0.001$ \\
\hline Location (Left) & $\mathrm{I} .467$ & $0.996-2.160$ & 0.052 & \\
\hline Size & 0.989 & $0.968-1.012$ & 0.347 & \\
\hline Organ invasion (Yes) & 0.493 & $0.257-0.947$ & 0.034 & 0.009 \\
\hline Histologic subtypes & & & $<0.001$ & \\
\hline DD VS WD & 1.050 & $0.252-4.374$ & 0.947 & \\
\hline Myxoid/Round Cell VS WD & 2.631 & $0.642-10.787$ & 0.179 & \\
\hline Pleomorphic VS WD & 1.964 & $0.443-8.705$ & 0.375 & \\
\hline FNCLCC & & & $<0.001$ & \\
\hline Grade 2 VS Grade I & 0.342 & $0.203-0.573$ & $<0.001$ & 0.042 \\
\hline Grade 3 VS Grade I & 0.492 & $0.32 I-0.755$ & 0.001 & $<0.001$ \\
\hline Number of resected organs $(\leq 1)$ & 1.197 & $0.812-1.765$ & 0.363 & \\
\hline
\end{tabular}


Points

Gender

Age

Presentation status

FNCLCC Grade

Organ_invasion

Total Points

1 year DFS rate

2 years DFS rate

5 years DFS rate

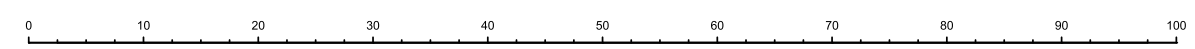

Male

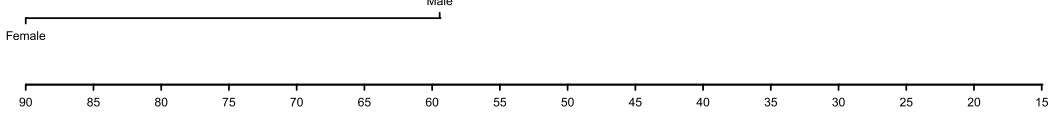

Recurrence

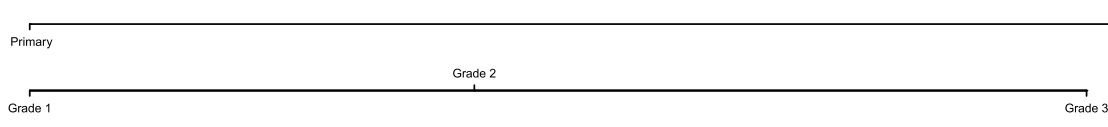

Yes

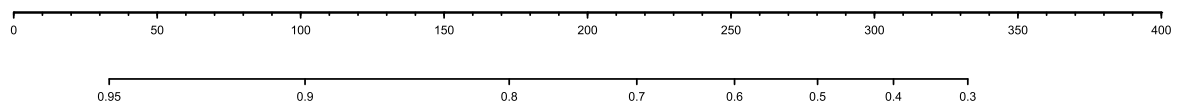

\begin{tabular}{rrrrrr}
\hline 0.9 & 1 & 1 & & & \\
0.8 & 0.7 & 0.6 & 0.5 & 0.4 & 0.3
\end{tabular}

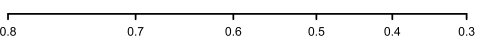

Figure I Nomogram for 1-, 2- and 5-year recurrence-free survival in patients with retroperitoneal liposarcoma after surgery.
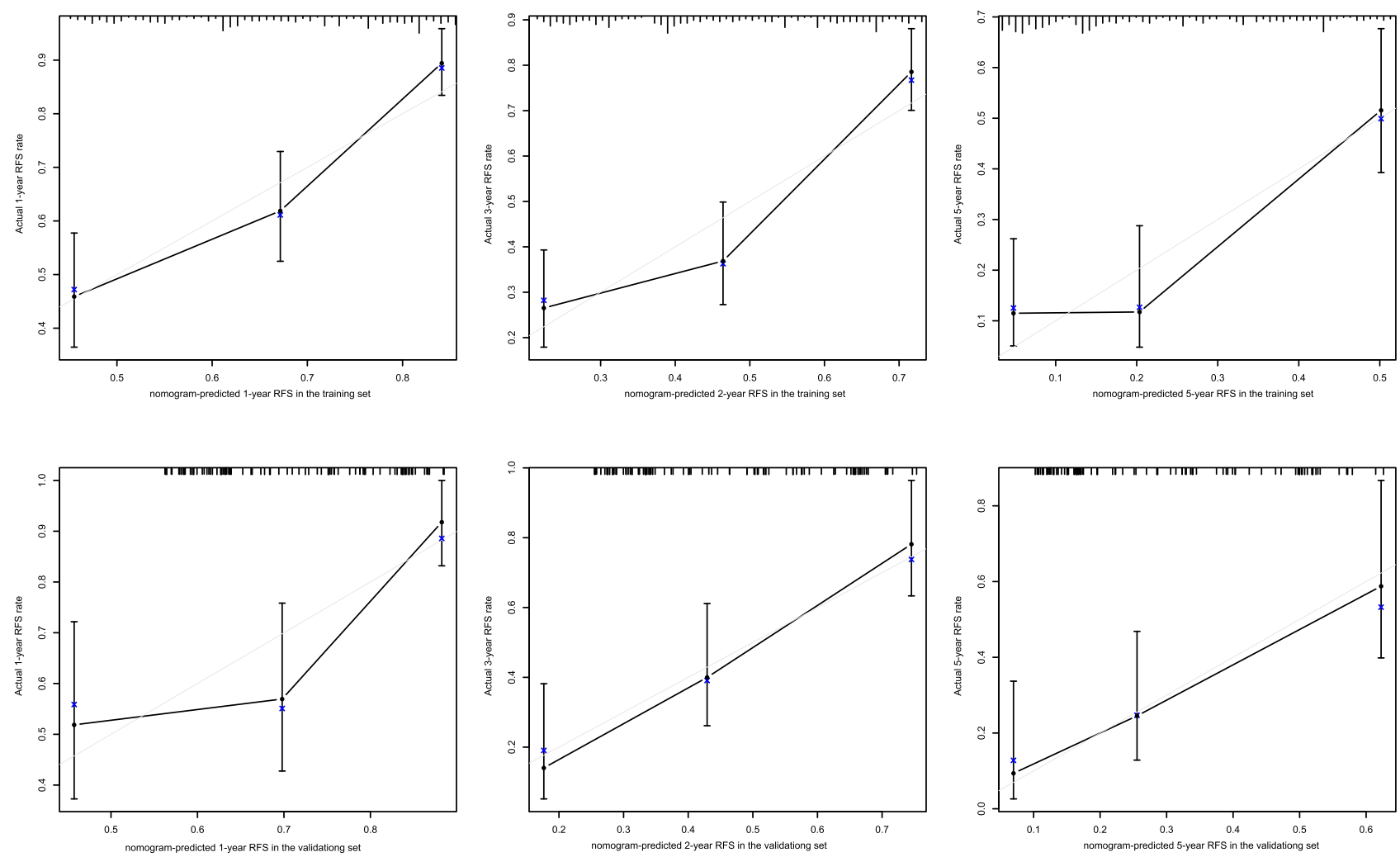

Figure 2 Calibration plots for predicting I-, 2- and 5-year recurrence-free survival in the training and test sets. X-axis: bootstrap-predicted survival; $y$-axis: actual outcome.

A separate nomogram for RLPS appeared in 2020, Xue et $\mathrm{al}^{6}$ which included 124 patients with RLPS. They defined the endpoint as local recurrence-free survival, with 3-year and 5-year local recurrence-free survival rates were $35.6 \%$ and $28.2 \%$. This nomogram includes five variables of presentation status (primary or recurrence), multifocality, completeness of resection, histologic subtypes, and FNCLCC grade. In the absence of external validation, the concordance index was 0.732 . This is basically the first nomogram we found that enrolled RPLS. The cons of the nomogram are that due to restricted tumour conditions fewer patients were included. Thus, there is no external validation. On the contrary, we divided 447 RLPS patients into training and test set, modeled and 

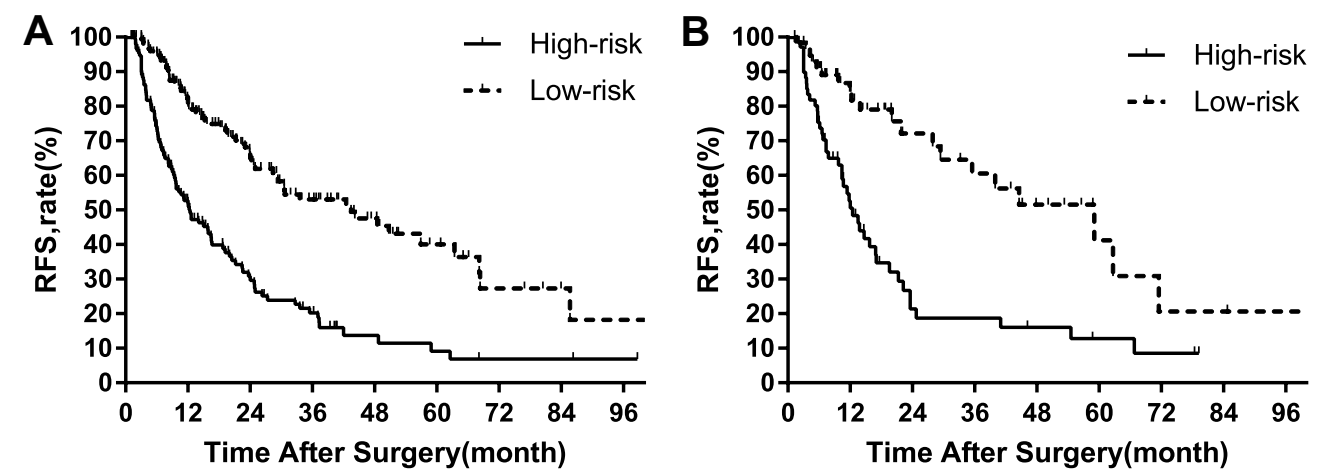

Figure 3 Kaplan-Meier curves of recurrence-free survival for patients with retroperitoneal liposarcoma after surgery in the high- and low-risk groups. (A) Training set; (B) test set.

verified. With larger patients pool, we firmly believe that our tool yields better predictions. Furthermore, retroperitoneal liposarcoma owns different biological behaviors and treatment strategies between Asian and Western populations. ${ }^{15}$ However, the current studies on Asian populations are few as well as the sample size is small. In a country like China with a large area, a large population, and a high concentration of medical resources, rare diseases, such as a retroperitoneal liposarcoma, the nomogram established by using the data advantages of the highvolume center and the treatment experience can guide and help other regions' treatment options and patient consultation in Asia.

Hence, this study took advantage of the high-volume sarcoma reference centre of South Hospital of Zhongshan Hospital/Shanghai Public Health Clinical Center, analyzed 447 cases of retroperitoneal liposarcoma patients and developed a nomogram prediction model to estimate 1-, 2- and 5-year RFS rate. Discrimination and calibration analyses were employed to evaluate the performance of our nomogram. Results showed that our nomogram was an effective and accurate model. The patients were further stratified into high- and low-risk groups for postoperative recurrence through the nomogram prediction model. The high-risk group has a significantly higher risk of recurrence, and there are significant statistical differences between the two groups.

In this study, the younger age gets higher score, which implies the higher the risk of recurrence. The result is consistent with previous reports conducted by other authors. ${ }^{16}$ In terms of gender, male means a higher rate of disease recurrence, which is also similar to previous reports. ${ }^{17}$ We also show that the impact of nearby organ invasion on the prognosis of RLPS. Surgeons usually take RLPS as an impermeable pressure mass. Nonetheless, we show that $84.8 \%$ of RLPS with one or more organs removed due to infiltrated. Sometimes, RLPS is indistinguishable from normal adipose tissue by the naked eye, as well as difficult to identify the permeability pattern or multifocality. Previous studies have confirmed that FNCLCC grade is related to the prognosis of retroperitoneal tumors, ${ }^{18,19}$ consistent with the conclusions of this research. Our work demonstrates the importance of the relationship between performance status and RFS rates. Each recurrence of the disease will result in a lower complete surgical resection rate and RFS rate. ${ }^{20,21}$ For recurrent diseases, whether the initial treatment is in the high-flow sarcoma diagnosis and treatment center is no longer an independent prognostic risk factor. ${ }^{20}$ This also emphasizes the importance of the first high-quality surgical treatment.

This retrospective study has several limitations. First, it is a retrospective study with all study cases from the same center and external validation is needed. Secondly, a few patients were excluded due to incomplete data.

In summary, we build a nomogram to evaluate the RFS in Asian patients with surgically resected RLPS. This makes up for the lack of research related to retroperitoneal liposarcoma in Asian population.

\section{Data Sharing Statement}

The data that support the findings of this study are available from the corresponding authors upon reasonable request.

\section{Ethics Statement}

This study was approved by the institutional review board of South Hospital of the Zhongshan Hospital/Shanghai 
Public Health Clinical Center (Shanghai, China), and was carried out following the Declaration of Helsinki. All patients provided written informed consent.

\section{Disclosure}

The authors confirm that there are no conflicts of interest.

\section{References}

1. Lee A, Thway K, Huang PH, Jones RL. Clinical and molecular spectrum of liposarcoma. J Clin Oncol. 2018;36(2):151-159. doi:10.1200/JCO.2017.74.9598

2. De Vita A, Mercatali L, Recine F, et al. Current classification, treatment options, and new perspectives in the management of adipocytic sarcomas. Onco Targets Ther. 2016;9:6233-6246. doi:10.2147/OTT. S112580

3. Vijay A, Ram L. Retroperitoneal liposarcoma: a comprehensive review. Am J Clin Oncol. 2015;38(2):213-219. doi:10.1097/ COC.0b013e31829b5667

4. Dalal KM, Kattan MW, Antonescu CR, Brennan MF, Singer S. Subtype specific prognostic nomogram for patients with primary liposarcoma of the retroperitoneum, extremity, or trunk. Ann Surg. 2006;244(3):381-391. doi:10.1097/01.sla.0000234795.98607.00

5. Gronchi A, Miceli R, Shurell E, et al. Outcome prediction in primary resected retroperitoneal soft tissue sarcoma: histology-specific overall survival and disease-free survival nomograms built on major sarcoma center data sets. J Clin Oncol. 2013;31(13):1649-1655. doi:10.1200/ JCO.2012.44.3747

6. Xue G, Wang Z, Li C, et al. A novel nomogram for predicting local recurrence-free survival after surgical resection for retroperitoneal liposarcoma from a Chinese tertiary cancer center. Int J Clin Oncol. 2021;26(1):145-153. doi:10.1007/s10147-020-01796-6

7. Gronchi A, Strauss DC, Miceli R, et al. Variability in Patterns of recurrence after resection of primary retroperitoneal sarcoma (RPS): a report on 1007 patients from the multi-institutional collaborative rps working group. Ann Surg. 2016;263(5):1002-1009. doi:10.1097/ SLA.0000000000001447

8. Fletcher CDM, Bridge JA, Hogendoorn PCW, Mertens F, editors. WHO Classification of Tumours of Soft Tissue and Bone. Pathology and Genetics of Tumours of Soft Tissue and Bone. 4th ed. Lyon: IARC Press; 2013.

9. Akaike H. A new look at the statistical model identification. IEEE Trans Autom Control. 1974;19(6):716-723. doi:10.1109/ TAC.1974.1100705
10. Strauss DC, Hayes AJ, Thway K, Moskovic EC, Fisher C, Thomas JM. Surgical management of primary retroperitoneal sarcoma. Br J Surg. 2010;97(5):698-706. doi:10.1002/bjs.6994

11. Tan MC, Brennan MF, Kuk D, et al. Histology-based classification predicts pattern of recurrence and improves risk stratification in primary retroperitoneal sarcoma. Ann Surg. 2016;263(3):593-600. doi:10.1097/SLA.0000000000001149

12. Ardoino I, Miceli R, Berselli M, et al. Histology-specific nomogram for primary retroperitoneal soft tissue sarcoma. Am Cancer Soc. 2010;116(10):2429-2436.

13. Chou YS, Liu CY, Chang YH, et al. Prognostic factors of primary resected retroperitoneal soft tissue sarcoma: analysis from a single asian tertiary center and external validation of gronchi's nomogram. J Surg Oncol. 2016;113(4):355-360. doi:10.1002/jso.24155

14. Raut CP, Miceli R, Strauss DC, et al. External validation of a multi-institutional retroperitoneal sarcoma nomogram. Am Cancer Soc. 2016;122(9):1417-1424.

15. Wong RX, Koh YS, Ong F, Farid M, Tay T, Teo M. Applicability of the sarculator and MSKCC nomograms to retroperitoneal sarcoma prognostication in an Asian tertiary center. Asian J Surg. 2020;43 (11):1078-1085. doi:10.1016/j.asjsur.2020.01.005

16. Nathan H, Raut CP, Thornton K, et al. Predictors of survival after resection of retroperitoneal sarcoma: a population-based analysis and critical appraisal of the AJCC staging system. Ann Surg. 2009;250 (6):970-976. doi:10.1097/SLA.0b013e3181b25183

17. Canter RJ, Beal S, Borys D, Martinez SR, Bold RJ, Robbins AS. Interaction of histologic subtype and histologic grade in predicting survival for soft-tissue sarcomas. J Am Coll Surg. 2010;210 (2):191-198. doi:10.1016/j.jamcollsurg.2009.10.007

18. Bonvalot S, Rivoire M, Castaing M, et al. Primary retroperitoneal sarcomas: a multivariate analysis of surgical factors associated with local control. J Clin Oncol. 2009;27(1):31-37. doi:10.1200/ JCO.2008.18.0802

19. Gronchi A, Lo VS, Fiore M, et al. Aggressive surgical policies in a retrospectively reviewed single-institution case series of retroperitoneal soft tissue sarcoma patients. J Clin Oncol. 2009;27(1):24-30. doi:10.1200/JCO.2008.17.8871

20. Lewis JJ, Leung D, Woodruff JM, Brennan MF. Retroperitoneal soft-tissue sarcoma: analysis of 500 patients treated and followed at a single institution. Ann Surg. 1998;228(3):355-365. doi:10.1097/ 00000658-199809000-00008

21. Grobmyer SR, Wilson JP, Apel B, et al. Recurrent retroperitoneal sarcoma: impact of biology and therapy on outcomes. $\mathrm{J} \mathrm{Am} \mathrm{Coll}$ Surg. 2010;210(5):602-608, 608-610. doi:10.1016/j. jamcollsurg.2009.12.024
Cancer Management and Research

\section{Publish your work in this journal}

Cancer Management and Research is an international, peer-reviewed open access journal focusing on cancer research and the optimal use of preventative and integrated treatment interventions to achieve improved outcomes, enhanced survival and quality of life for the cancer patient.
The manuscript management system is completely online and includes a very quick and fair peer-review system, which is all easy to use. Visit http://www.dovepress.com/testimonials.php to read real quotes from published authors. 